\title{
Semi-invariants of Binary Forms Pertaining to a Unimodality Theorem of Reiner and Stanton
}

\author{
William Y.C. Chen ${ }^{1}$ and Ivy D.D. Jia ${ }^{2}$ \\ ${ }^{1,2}$ Chern Institute of Mathematics \\ Nankai University \\ Tianjin 300071, P. R. China \\ and \\ ${ }^{1}$ Center for Applied Mathematics \\ Tianjin University \\ Tianjin 300072, P. R. China \\ Emails: ${ }^{1}$ chenyc@tju.edu.cn, ${ }^{2}$ jiadandan@ mail.nankai.edu.cn \\ Dedicated to the Memory of Professor S. S. Chern
}

\begin{abstract}
The symmetric difference of the $q$-binomial coefficients $F_{n, k}(q)=\left[\begin{array}{c}n+k \\ k\end{array}\right]-$ $q^{n}\left[\begin{array}{c}n+k-2 \\ k-2\end{array}\right]$ was introduced by Reiner and Stanton. They proved that $F_{n, k}(q)$ is symmetric and unimodal for $k \geq 2$ and $n$ even by using the representation theory for Lie algebras. Based on Sylvester's proof of the unimodality of the Gaussian coefficients, as conjectured by Cayley, we find an interpretation of the unimodality of $F_{n, k}(q)$ in terms of semi-invariants. In the spirit of the strict unimodality of the Gaussian coefficients due to Pak and Panova, we prove the strict unimodality of the symmetric difference $G_{n, k, r}(q)=\left[\begin{array}{c}n+k \\ k\end{array}\right]-q^{n r / 2}\left[\begin{array}{c}n+k-r \\ k-r\end{array}\right]$, except for the two terms at both ends, where $n, r \geq 8, k \geq r$ and at least one of $n$ and $r$ is even.
\end{abstract}

AMS Classification: 05A17, 05E10, 13A50

Keywords: semi-invariants, binary forms, Gaussian coefficients, partitions, unimodality

\section{Introduction}

The story begins with the Gaussian coefficients called by Rota with no particular reasons (private conversation, see also [9]), or sometimes the Gaussian polynomials, or often under the name of the $q$-binomial coefficients, as given by

$$
\left[\begin{array}{l}
n \\
k
\end{array}\right]=\frac{\left(1-q^{n}\right)\left(1-q^{n-1}\right) \cdots\left(1-q^{n-k+1}\right)}{\left(1-q^{k}\right)\left(1-q^{k-1}\right) \cdots(1-q)},
$$

where $0 \leq k \leq n$. The Gaussian coefficients are polynomials in $q$ and they enjoy the fundamental property that their coefficients are symmetric and unimodal, as conjectured 
by Cayley [4] in 1856 and confirmed by Sylvester [30] in 1878, who had even believed that settling the conjecture of Cayley was a task that lay outside the human power, see also, Pak and Panova [15,16]. Ever since a great deal of work has been done in this vein, see, for example, [3, 6-8, 10, 13, 14, 17-21, 24, 25, 28, 31,33], to mention only a few. In particular, O'Hara [14] found a combinatorial proof, Zeilberger [33] came up with an identity, known as the $\mathrm{KOH}$ theorem, which serves the purpose of justifying the unimodality.

The unimodality is not only associated with the Gaussian coefficients, it can also be said about certain differences of the Gaussian coefficients. Employing the representation theory for Lie algebras, Reiner and Stanton [22] established the unimodality of

$$
\left[\begin{array}{l}
n \\
k
\end{array}\right]-\left[\begin{array}{c}
n \\
k-1
\end{array}\right],
$$

where $k \geq 1, n$ is odd and $2 k \leq n+1$. They also showed that

$$
F_{n, k}(q)=\left[\begin{array}{c}
n+k \\
k
\end{array}\right]-q^{n}\left[\begin{array}{c}
n+k-2 \\
k-2
\end{array}\right]
$$

is symmetric and unimodal when $k \geq 2$ and $n$ is even. Furthermore, Reiner and Stanton conjectured the unimodality of

$$
\left[\begin{array}{c}
n-1 \\
k
\end{array}\right]-q^{n-2 r k+1+4(r-1)}\left[\begin{array}{c}
n-1+4(r-1) \\
k-2
\end{array}\right],
$$

where $n$ is odd, $k \geq 2, r \geq 1$ and $n \geq 2 r k-4 r+3$. This conjecture is still open.

The above symmetric differences (1.3) are called the strange symmetric differences by Stanley and Zanello [27]. They extended the above conjecture to a broader framework, namely, for each $k \geq 5$, the polynomials

$$
f_{k, m, b}(q)=\left[\begin{array}{c}
m \\
k
\end{array}\right]-q^{\frac{k(m-b)}{2}+b-2 k+2}\left[\begin{array}{c}
b \\
k-2
\end{array}\right]
$$

are nonnegative and unimodal subject to certain conditions. They verified their conjecture for $2 \leq k \leq 5$ by means of the $\mathrm{KOH}$ theorem of Zeilberger [33]. Notice that when $b=m-2,(1.4)$ reduces to (1.2).

It is worth mentioning that Bergeron [2] investigated the symmetric differences of the following form:

$$
\left[\begin{array}{c}
b+c \\
b
\end{array}\right]-\left[\begin{array}{c}
a+d \\
d
\end{array}\right]
$$

where $a, b, c, d$ are positive integers, with $a$ being the smallest and $a d=b c$. It was conjectured that such symmetric differences are polynomials in $q$ with nonnegative coefficients. Zanello [32] further conjectured the unimodality of (1.5), and proved the unimodality for $a \leq 3$ and $b, c \geq 4$, resorting to the $\mathrm{KOH}$ theorem.

A notable progress on the unimodality of the Gaussian coefficients was achieved by Pak and Panova $[15,16]$. They showed that the Gaussian coefficients are strictly unimodal 
except for a few cases. Recall that a polynomial $f(x)=a_{0}+a_{1} x+a_{2} x^{2}+\cdots+a_{n} x^{n}$ with positive coefficients is said to be unimodal if there is an index $m$ such that

$$
a_{0} \leq a_{1} \leq \cdots \leq a_{m} \geq a_{m+1} \geq \cdots \geq a_{n} .
$$

We say that $f(x)$ is strictly unimodal if (1.6) is to be replaced by

$$
a_{0}<a_{1}<\cdots<a_{m}>a_{m+1}>\cdots>a_{n}
$$

The humble goal of this paper is to present an interpretation of the unimodality of $F_{n, k}(q)$ as in (1.2) in terms of semi-invariants of binary forms, following the original aspiration of Sylvester. Once the connection to semi-invariants is at our disposal, we carry on to prove the strict unimodality of the symmetric difference

$$
G_{n, k, r}(q)=\left[\begin{array}{c}
n+k \\
k
\end{array}\right]-q^{\frac{n r}{2}}\left[\begin{array}{c}
n+k-r \\
k-r
\end{array}\right],
$$

except for the two terms at both ends, when $n, r \geq 8, k \geq r$ and at least one of $n$ and $r$ is even.

It is our hope that after a sound rest for more than a century, it might be the time for the once shining invariant theory of binary forms to shed light on the ongoing study of the theory of partitions, presumably under a banner bearing the name of enumerative invariant theory.

\section{Semi-invariants}

A binary form of degree $n$, or a binary $n$-form, is a homogeneous polynomial in $x$ and $y$,

$$
f(x, y)=a_{0} x^{n}+\left(\begin{array}{l}
n \\
1
\end{array}\right) a_{1} x^{n-1} y+\left(\begin{array}{l}
n \\
2
\end{array}\right) a_{2} x^{n-2} y^{2}+\cdots+a_{n} y^{n} .
$$

A semi-invariant or a source of a covariant, called by Cayley [5], or a differentiant called by Sylvester [30], of the binary form $f(x, y)$, is a polynomial $I\left(a_{0}, a_{1}, \ldots, a_{n}\right)$ in $a_{0}, a_{1}, \ldots, a_{n}$ with rational coefficients that is invariant under the transformation:

$$
\left(\begin{array}{l}
x \\
y
\end{array}\right)=\left(\begin{array}{ll}
1 & h \\
0 & 1
\end{array}\right)\left(\begin{array}{l}
x^{\prime} \\
y^{\prime}
\end{array}\right)
$$

that is, $x=x^{\prime}+h y^{\prime}$ and $y=y^{\prime}$. The matrix in (2.2) is called a shear matrix. Notice that Hilbert [12] used the Greek letter $\mu$ in place of $h$ in the shear matrix in (2.2). Here we prefer the above notation of Sylvester [30] for the reason that the Greek letters $\lambda, \mu$ are now often reserved for integer partitions. Indeed, the transformation (2.2) for binary forms goes back to Lagrange, see [11,23]. More precisely, suppose that the binary form (2.1) becomes

$$
f^{\prime}\left(x^{\prime}, y^{\prime}\right)=a_{0}^{\prime} x^{\prime n}+\left(\begin{array}{l}
n \\
1
\end{array}\right) a_{1}^{\prime} x^{\prime n-1} y^{\prime}+\left(\begin{array}{l}
n \\
2
\end{array}\right) a_{2}^{\prime} x^{\prime n-2} y^{\prime 2}+\cdots+a_{n}^{\prime} y^{\prime n}
$$


under the transformation (2.2), where, for $0 \leq i \leq n$,

$$
a_{i}^{\prime}=a_{i}+\left(\begin{array}{l}
i \\
1
\end{array}\right) a_{i-1} h+\left(\begin{array}{l}
i \\
2
\end{array}\right) a_{i-2} h^{2}+\cdots+a_{0} h^{i} .
$$

Then we say that a polynomial $I\left(a_{0}, a_{1}, \ldots, a_{n}\right)$ with rational coefficients is a semiinvariant of the binary form (2.1) if

$$
I\left(a_{0}, a_{1}, \ldots, a_{n}\right)=I\left(a_{0}^{\prime}, a_{1}^{\prime}, \ldots, a_{n}^{\prime}\right)
$$

for any shear transformation in (2.2).

For example, for the quadratic form

$$
f(x, y)=a_{0} x^{2}+2 a_{1} x y+a_{2} y^{2},
$$

it can be easily checked that the polynomial

$$
I\left(a_{0}, a_{1}, a_{2}\right)=a_{0} a_{2}-a_{1}^{2}
$$

is a semi-invariant. In fact, it is the discriminant, which is more than a semi-invariant in the sense that it is an invariant with respect to a more general transformation, see, for example, [11,29]. Below is a semi-invariant but not an invariant:

$$
J\left(a_{0}, a_{1}, a_{2}\right)=a_{0}^{2} a_{2}-a_{0} a_{1}^{2} .
$$

As far as this paper is concerned, a semi-invariant can be viewed as nothing but a polynomial $I\left(a_{0}, a_{1}, \ldots, a_{n}\right)$ that satisfies a partial differential equation. Needless to say, the coefficients $a_{0}, a_{1}, \ldots, a_{n}$ are perceived as variables. First, the degree of a monomial

$$
a^{\nu}=a_{0}^{\nu_{0}} a_{1}^{\nu_{1}} \cdots a_{n}^{\nu_{n}}
$$

is meant to be the total degree $\nu_{0}+\nu_{1}+\cdots+\nu_{n}$. Besides, the weight of the monomial $a^{\nu}$ is defined by

$$
\nu_{1}+2 \nu_{2}+\cdots+n \nu_{n}
$$

For the purpose of this paper, we shall only be concerned with homogeneous semiinvariants with respect to the degree and the weight. For example, the semi-invariant $I\left(a_{0}, a_{1}, a_{2}\right)$ in (2.7) has degree two and weight two, whereas the semi-invariant $J\left(a_{0}, a_{1}, a_{2}\right)$ in (2.8) has degree three and weight two.

We are now led to define $Q_{n}(k, m)$ as the vector space of polynomials in $a_{0}, a_{1}, \ldots, a_{n}$ over the rational numbers that are homogeneous of degree $k$ and weight $m$. Taking the dimension of $Q_{n}(k, m)$ into account, the notion of partitions immediately comes into play. The monomials in $Q_{n}(k, m)$ are in one-to-one correspondence with partitions of $m$ into $k$ parts with zero parts allowed and each part not exceeding $n$. Recall that a partition of $m$ with $k$ parts such that no parts exceed $n$ can be represented as

$$
\lambda=0^{\nu_{0}} 1^{\nu_{1}} \cdots n^{\nu_{n}},
$$


where $\nu_{i}$ signifies the number of occurrences of the part $i$ in $\lambda$ and $\nu_{0}+\nu_{1}+\cdots+\nu_{n}=k$. We say that such a partition is contained in a $k \times n$ rectangle. Clearly, $\lambda$ is a partition of $\nu_{1}+2 \nu_{2}+\cdots+n \nu_{n}$, which is the weight of the monomial $a^{\nu}$. This basic fact brings us right to the stage of the Gaussian coefficients which admit the following partition interpretation.

Let $p(k, n, m)$ denote the number of partitions of $m$ contained in a $k \times n$ rectangle, then we have

$$
\left[\begin{array}{c}
n+k \\
k
\end{array}\right]=\sum_{m=0}^{n k} p(k, n, m) q^{m},
$$

see $[1,26]$. The above polynomial is symmetric, that is, for $0 \leq m \leq n k / 2$,

$$
p(k, n, m)=p(k, n, n k-m) .
$$

For $0 \leq m \leq n k / 2$, Sylvester found an interpretation of the difference $p(k, n, m)-$ $p(k, n, m-1)$ in terms of semi-invariants, which demystifies the unimodality of the Gaussian coefficients.

The following characterization is crucial for the route from the vector space $Q_{n}(k, m)$ to semi-invariants, see Cayley [4] or Hilbert [12].

Theorem 2.1. Let

$$
D=a_{0} \frac{\partial}{\partial a_{1}}+2 a_{1} \frac{\partial}{\partial a_{2}}+3 a_{2} \frac{\partial}{\partial a_{3}}+\cdots+n a_{n-1} \frac{\partial}{\partial a_{n}} .
$$

A polynomial $I\left(a_{0}, a_{1}, \ldots, a_{n}\right)$ in $Q_{n}(k, m)$ is a semi-invariant of the binary form (2.1) if and only if $D I\left(a_{0}, a_{1}, \ldots, a_{n}\right)=0$.

Taking the semi-invariant $J\left(a_{0}, a_{1}, a_{2}\right)$ in (2.8) as an example, it can be seen that

$$
D J\left(a_{0}, a_{1}, a_{2}\right)=\left(a_{0} \frac{\partial}{\partial a_{1}}+2 a_{1} \frac{\partial}{\partial a_{2}}\right)\left(a_{0}^{2} a_{2}-a_{0} a_{1}^{2}\right)
$$

is identically zero.

Let $S_{n}(k, m)$ denote the set of semi-invariants of degree $k$ and weight $m$, that is,

$$
S_{n}(k, m)=\left\{I \in Q_{n}(k, m) \mid D(I)=0\right\},
$$

where $D$ is the linear operator defined in (2.14). Bear in mind that $S_{n}(k, m)$ forms a vector space over $\mathbb{Q}$. By the number of semi-invariants of degree $k$ and weight $m$ of a binary $n$-form, we really mean the dimension of the vector space $S_{n}(k, m)$. For example, $\operatorname{dim} S_{4}(4,6)=2$. Below are two linearly independent semi-invariants of degree four and weight six of a binary 4-form:

$$
\begin{aligned}
& I_{1}=3 a_{1}^{2} a_{2}^{2}-4 a_{1}^{3} a_{3}-2 a_{0} a_{1} a_{2} a_{3}+3 a_{0}^{2} a_{3}^{2}+4 a_{0} a_{1}^{2} a_{4}-4 a_{0}^{2} a_{2} a_{4}, \\
& I_{2}=a_{0} a_{2}^{3}-2 a_{0} a_{1} a_{2} a_{3}+a_{0}^{2} a_{3}^{2}+a_{0} a_{1}^{2} a_{4}-a_{0}^{2} a_{2} a_{4} .
\end{aligned}
$$

We now come to the remarkable discovery of Sylvester [30]. 
Theorem 2.2. For $n, k \geq 0$ and $0 \leq m \leq n k / 2$, the number of semi-invariants of $a$ binary $n$-form of degree $k$ and weight $m$ equals

$$
\delta(k, n, m)=p(k, n, m)-p(k, n, m-1),
$$

with the convention that $p(k, n,-1)=0$.

For example, for the quadratic form

$$
f(x, y)=a_{0} x^{2}+2 a_{1} x y+a_{2} y^{2},
$$

there is exactly one semi-invariant of degree three and weight two, that is,

$$
J\left(a_{0}, a_{1}, a_{2}\right)=a_{0}^{2} a_{2}-a_{0} a_{1}^{2} .
$$

On the other hand, we have $p(3,2,2)=2$ and $p(3,2,1)=1$, in accordance with Theorem 2.2 .

Up to now, we are sufficiently equipped to move on to the next section to explore symmetric differences of the Gaussian coefficients by means of semi-invariants.

\section{Symmetric Differences of the Gaussian Coefficients}

The first objective of this section is to give a semi-invariant interpretation of the unimodality theorem of Reiner and Stanton on $F_{n, k}(q)$ as in (1.2). Then we proceed to prove the strict unimodality of $G_{n, k, r}(q)$ as in (1.8).

Lemma 3.1. If $n$ is even, then there is exactly one semi-invariant of a binary $n$-form of degree two and weight $n$.

Proof. It is not hard to see that for $m \leq n$,

$$
p(2, n, m)=\left\lfloor\frac{m+2}{2}\right\rfloor,
$$

see Stanley and Zanello [27,32]. Since $n$ is even, by Theorem 2.2 we see that there is only one semi-invariant of degree two and weight $n$.

For example, there is only one semi-invariant of a binary 4-form of degree two and weight four, that is,

$$
J=3 a_{2}^{2}-4 a_{1} a_{3}+a_{0} a_{4}
$$

The argument in the proof of Lemma 3.1 indicates that when $n$ is odd, there are no semi-invariants of a binary $n$-form of degree two and weight $n$. The following relation is essentially a consequence of the fact that the set of semi-invariants forms a ring. To be precise, if $I$ and $J$ are two semi-invariants of a binary $n$-form, then so are $I+J$ and $I J$. In fact, the ring property of semi-invariants is a consequence of the operator characterization as stated in Theorem 2.1. 
Theorem 3.2. If $k \geq 2, m \geq n$ and $n$ is even, then the number of semi-invariants of a binary $n$-form of degree $k$ and weight $m$ is at least the number of semi-invariants of degree $k-2$ and weight $m-n$, that is,

$$
\delta(k, n, m) \geq \delta(k-2, n, m-n) .
$$

Proof. Assume that there are $t$ linearly independent semi-invariants of degree $k-2$ and weight $m-n$, say, $I_{1}, I_{2}, \ldots, I_{t}$, where the variables $a_{0}, a_{1}, \ldots, a_{n}$ are suppressed. We wish to show that there are at least $t$ semi-invariants of degree $k$ and weight $m$. By Lemma 3.1 , we may assume that $J$ is a semi-invariant of a binary $n$-form of degree two and weight $n$. Thanks to the ring structure of semi-invariants, we see that $J I_{1}, J I_{2}, \ldots, J I_{t}$ are semiinvariants of degree $k$ and weight $m$. To complete the proof, one only needs to realize that $J I_{1}, J I_{2}, \ldots, J I_{t}$ are linearly independent, which is by any means a plain fact.

Next we demonstrate that Reiner and Stanton's unimodality theorem for $F_{n, k}(q)$ is immediate from Theorem 3.2. Recall that

$$
F_{n, k}(q)=\left[\begin{array}{c}
n+k \\
k
\end{array}\right]-q^{n}\left[\begin{array}{c}
n+k-2 \\
k-2
\end{array}\right],
$$

where $k \geq 2$ and $n$ is even.

While keeping the symmetry of $F_{n, k}(q)$ in mind, to confirm the unimodality, let

$$
F_{n, k}(q)=\sum_{m=0}^{n k} f_{m} q^{m}
$$

so that

$$
f_{m}=p(k, n, m)-p(k-2, n, m-n),
$$

with the convention that $p(k-2, n, j)=0$ if $j$ is negative. For $0 \leq m \leq n k / 2$, in light of Theorem 2.2, the inequality (3.3) yields

$$
p(k, n, m)-p(k, n, m-1) \geq p(k-2, n, m-n)-p(k-2, n, m-n-1),
$$

which can be recast as

$$
p(k, n, m)-p(k-2, n, m-n) \geq p(k, n, m-1)-p(k-2, n, m-n-1) .
$$

But this is exactly $f_{m} \geq f_{m-1}$.

Notice that $F_{n, k}(q)$ is not always strictly unimodal, except for the two terms at both ends. For example, for $F_{5,9}(q)$ and $F_{14,5}(q)$, the maximal coefficients occur more than twice in the middle. The following conjecture is supported by numerical evidence.

Conjecture 3.3. For $n \geq 8$ and $k \geq 15, F_{n, k}(q)$ is strictly unimodal, except for the two terms at both ends.

One might expect to push forward along this direction to tackle the strict unimodality of $F_{n, k}(q)$. But it is not clear as to how this can be pursued. Nevertheless, we are given a chance to establish the strict unimodality of $G_{n, k, r}(q)$, with the two terms at the beginning and at the end being excluded, to be precise. To this end, we need two special semiinvariants, as ensured by the following lemma. 
Lemma 3.4. If $n, r \geq 8$ and at least one of $n$ and $r$ is even, then there are at least two linearly independent semi-invariants of a binary $n$-form of degree $r$ and weight $n \mathrm{r} / 2$, that is,

$$
\delta(r, n, n r / 2) \geq 2
$$

In a more general setting, Pak and Panova $[15,16]$ obtained the strict unimodality of the Gaussian coefficients.

Theorem 3.5. For all $n, k \geq 8$ and $2 \leq m \leq n k / 2$, we have $\delta(k, n, m) \geq 1$.

It should be pointed out that the following proof of Lemma 3.4 is reminiscent of the reduction argument of Pak and Panova.

Proof of Lemma 3.4. For $8 \leq n, r<16$, it is easily verified that (3.9) holds. We now turn to the case $8 \leq n<16$ and $r \geq 16$. Write $r=8 s+t$, where $s \geq 1$ and $8 \leq t<16$. As is known for the first case, we have $\delta(8, n, 4 n) \geq 2$. This means that there exists a semiinvariant $I$, with the variables being suppressed, of a binary $n$-form of degree eight and weight $4 n$. Again, the argument for the first case implies $\delta(t, n, n t / 2) \geq 2$. This enables us to find two linearly independent semi-invariants $J_{1}, J_{2}$ of a binary $n$-form of degree $t$ and weight $n t / 2$. Observe that $I^{s} J_{1}, I^{s} J_{2}$ are linearly independent semi-invariants of a binary $n$-form of degree $r$ and weight $n r / 2$, from which we obtain that $\delta(r, n, n r / 2) \geq 2$ for $8 \leq n<16$ and $r \geq 16$. Together with the first case $8 \leq n, r<16$, we see that $\delta(r, n, n r / 2) \geq 2$ for $8 \leq n<16$ and $r \geq 8$. Since

$$
p(r, n, n r / 2)=p(n, r, n r / 2),
$$

$\delta(r, n, n r / 2) \geq 2$ also holds for $n \geq 8$ and $8 \leq r<16$.

Now we are left only with the case $n \geq 8$ and $r \geq 16$. For $n \geq 8$, we write $r=8 s+t$, where $s \geq 1$ and $8 \leq t<16$. Mimicking the above reasoning for $8 \leq n<16$ and $r \geq 16$, we may reduce this case ( $n \geq 8$ and $r \geq 16$ ) back to the case $8 \leq n<16$ and $r \geq 16$. In summary, we conclude that $\delta(r, n, n r / 2) \geq 2$ holds for all $n, r \geq 8$.

Theorem 3.6. If $n, r \geq 8, k \geq r, m \geq n r / 2$, and at least one of $n$ and $r$ is even, then the number of semi-invariants of a binary $n$-form of degree $k$ and weight $m$ is greater than the number of semi-invariants of degree $k-r$ and weight $m-n r / 2$, that is,

$$
\delta(k, n, m)>\delta(k-r, n, m-n r / 2) .
$$

Let us see how the above relation yields the strict unimodality of $G_{n, k, r}(q)$.

Theorem 3.7. For $n, r \geq 8, k \geq r$ and at least one of $n$ and $r$ is even,

$$
G_{n, k, r}(q)=\left[\begin{array}{c}
n+k \\
k
\end{array}\right]-q^{\frac{n r}{2}}\left[\begin{array}{c}
n+k-r \\
k-r
\end{array}\right]
$$

is symmetric and strictly unimodal, except for the two terms at both ends. 
Proof. The symmetry of $G_{n, k, r}(q)$ can be easily verified. Let

$$
G_{n, k, r}(q)=\sum_{m=0}^{n k} g_{m} q^{m}
$$

and so

$$
g_{m}=p(k, n, m)-p(k-r, n, m-n r / 2),
$$

where we assume that $p(k-r, n, j)=0$ whenever $j$ is negative. Notice that $g_{0}=g_{1}=1$ and $g_{n k-1}=g_{n k}=1$. For $2 \leq m<n r / 2$, we have $g_{m}=p(k, n, m)$ since $p(k-r, n, m-$ $n r / 2)=0$. Thus, by Theorem 3.5 we see that $g_{m}-g_{m-1}=\delta(k, n, m)$, which is greater than or equal to one. For $n r / 2 \leq m \leq n k / 2$, in virtue of Theorem 2.2, the inequality (3.11) takes the form

$$
p(k, n, m)-p(k, n, m-1)>p(k-r, n, m-n r / 2)-p(k-r, n, m-n r / 2-1),
$$

which can be reformulated as $g_{m}>g_{m-1}$. Thus we have shown that $G_{n, k, r}(q)$ is symmetric and strictly unimodal.

For example, we have

$$
\begin{aligned}
G_{8,14,10}(q)=1+ & q+2 q^{2}+3 q^{3}+5 q^{4}+7 q^{5}+11 q^{6}+15 q^{7} \\
& +\cdots+8310 q^{53}+8408 q^{54}+8450 q^{55}+8479 q^{56} \\
& +8450 q^{57}+8408 q^{58}+8310 q^{59}+\cdots+11 q^{106} \\
& +7 q^{107}+5 q^{108}+3 q^{109}+2 q^{110}+q^{111}+q^{112}
\end{aligned}
$$

To present the proof of Theorem 3.6, it is necessary to define the leading term of a polynomial $f\left(a_{0}, a_{1}, \ldots, a_{n}\right)$. First, we write a monomial in the form $a^{\nu}=a_{0}^{\nu_{0}} a_{1}^{\nu_{1}} \cdots a_{n}^{\nu_{n}}$. Then we order the monomials of degree $k$ and weight $m$ according to the anti-lexicographic order of their exponents. Clearly, this order extends to the set of all monomials in $a_{0}, a_{1}, \ldots, a_{n}$. For example, for $k=4, n=4$ and $m=6$, we have

$$
a_{1}^{2} a_{2}^{2}>a_{0} a_{2}^{3}>a_{1}^{3} a_{3}>a_{0} a_{1} a_{2} a_{3}>a_{0}^{2} a_{3}^{2}>a_{0} a_{1}^{2} a_{4}>a_{0}^{2} a_{2} a_{4} .
$$

The leading term of a semi-invariant $I\left(a_{0}, a_{1}, \ldots, a_{n}\right)$, denoted by $\alpha\left(I\left(a_{0}, a_{1}, \ldots, a_{n}\right)\right)$ or $\alpha(I)$ for short, is defined to be the largest monomial with a nonzero coefficient with respect to the above order. For example, for the semi-invariants $I_{1}$ and $I_{2}$ in (2.17) and (2.18), we have

$$
\alpha\left(I_{1}\right)=a_{1}^{2} a_{2}^{2}, \quad \text { and } \quad \alpha\left(I_{2}\right)=a_{0} a_{2}^{3}
$$

Since the set of semi-invariants of a binary $n$-form of degree $k$ and weight $m$ forms a vector space, by a triangulation process or the Gauss elimination we may always find a set of semi-invariants whose leading terms are strictly decreasing with respect to the anti-lexicographic order.

We are now in a position to complete the proof of Theorem 3.6. 
Proof of Theorem 3.6. Let

$$
t=\delta(k-r, n, m-n r / 2)
$$

If $t=0$, by the strict unimodality of the Gaussian coefficients established by Pak and Panova $[15,16]$ as stated in Theorem 3.5 , we find that $\delta(k, n, m) \geq 1$. If $t>0$, we assume that $I_{1}, I_{2}, \ldots, I_{t}$, with the variables $a_{0}, a_{1}, \ldots, a_{n}$ being suppressed, are linearly independent semi-invariants of degree $k-r$ and weight $m-n r / 2$. By the triangulation process, we may further assume that the leading terms of $I_{1}, I_{2}, \ldots, I_{t}$ are strictly decreasing with respect to the anti-lexicographic order, that is,

$$
\alpha\left(I_{1}\right)>\alpha\left(I_{2}\right)>\cdots>\alpha\left(I_{t}\right)
$$

Next we attempt to construct $t+1$ linearly independent semi-invariants of degree $k$ and weight $m$. By Lemma 3.4, there exist two linearly independent semi-invariants $J_{1}$ and $J_{2}$ of a binary $n$-form of degree $r$ and weight $n r / 2$. Without loss of generality, let us assume that

$$
\alpha\left(J_{1}\right)>\alpha\left(J_{2}\right)
$$

We claim that $J_{1} I_{1}, J_{1} I_{2}, \ldots, J_{1} I_{t}, J_{2} I_{t}$ are linearly independent semi-invariants of degree $k$ and weight $m$. The degree and weight conditions are easily satisfied. It remains to verify that $J_{1} I_{1}, J_{1} I_{2}, \ldots, J_{1} I_{t}, J_{2} I_{t}$ are linearly independent. To this end, it suffices to show that the leading terms of $J_{1} I_{1}, J_{1} I_{2}, \ldots, J_{1} I_{t}, J_{2} I_{t}$ are distinct. For two semiinvariants $K_{1}$ and $K_{2}$, it is evident that

$$
\alpha\left(K_{1} K_{2}\right)=\alpha\left(K_{1}\right) \alpha\left(K_{2}\right)
$$

Under the assumption (3.16), we see that

$$
\alpha\left(J_{1} I_{1}\right)>\alpha\left(J_{1} I_{2}\right)>\cdots>\alpha\left(J_{1} I_{t}\right)
$$

Invoking the assumption (3.17), we get

$$
\alpha\left(J_{1} I_{t}\right)>\alpha\left(J_{2} I_{t}\right)
$$

Combining (3.19) and (3.20), we reach the conclusion that $J_{1} I_{1}, J_{1} I_{2}, \ldots, J_{1} I_{t}, J_{2} I_{t}$ have distinct leading terms, and hence they must be linearly independent. This completes the proof.

In fact, there is a slightly more general construction of semi-invariants, as stated below. The proof is omitted.

Lemma 3.8. Let $k_{1}, k_{2}, n \geq 0,0 \leq m_{1} \leq n k_{1} / 2,0 \leq m_{2} \leq n k_{2} / 2$. For a binary $n$-form, assume that there are $t_{1}$ linearly independent semi-invariants of degree $k_{1}$ and weight $m_{1}$, and $t_{2}$ linearly independent semi-invariants of degree $k_{2}$ and weight $m_{2}$, where $t_{1}, t_{2} \geq 1$. Then there exist $t_{1}+t_{2}-1$ linearly independent semi-invariants of degree $k_{1}+k_{2}$ and weight $m_{1}+m_{2}$. 
We conclude with a remark of Professor S. S. Chern. He once commented that GianCarlo Rota was fond of invariant theory, which he considered as a subject of old mathematics, in his own words, but not in a negative tone, to avoid any misunderstanding. In the same context, Professor Chern also mentioned that some papers of Gauss remain to be explored, but perhaps not easy to comprehend. As time passes, it may be witnessed that Rota had his reasons. At least it is our belief that this is something that should not completely slip our minds, or "there is something to it", as Rota would have put it.

Acknowledgment. This work was done under the auspices of the National Science Foundation of China.

\section{References}

[1] G.E. Andrews, The Theory of Partitions (Cambridge University Press, Cambridge, 1998).

[2] F. Bergeron, The $q$-Foulkes Conjecture (Talk delivered at Bowdoin College, ME, 2016).

[3] D.M. Bressoud, Unimodality of Gaussian polynomials, Discrete Math. 99 (1992), $17-24$.

[4] A. Cayley, A second memoir upon quantics, Philos. Trans. Roy. Soc. London 146 (1856), 101-126.

[5] A. Cayley, On the theorem of the finite number of the covariants of a binary quantic, Quart. J. Pure Appl. Math. 17 (1881), 137-147. Also in: The Collected Mathematical Papers of Arthur Cayley, Vol. XI, No. 761 (Cambridge University Press, Cambridge, 1896).

[6] V. Dhand, A combinatorial proof of strict unimodality for $q$-binomial coefficients, Discrete Math. 335 (2014), 20-24.

[7] J. Dousse and B. Kim, An overpartition analogue of the $q$-binomial coefficients, Ramanujan J. 42 (2017), 267-283.

[8] J. Dousse and B. Kim, An overpartition analogue of $q$-binomial coefficients, II: Combinatorial proofs and $(q, t)-\log$ concavity, J. Combin. Theory Ser. A 158 (2018), $228-253$.

[9] J. Goldman and G.-C. Rota, The number of subspaces of a vector space, Recent Progress in Combinatorics in Proc. Third Waterloo Conf. on Combinatorics, 1968 (Academic Press, New York, 1969), pp. 75-83.

[10] F.M. Goodman, K.M. O'Hara and D. Stanton, A unimodality identity for a Schur function, J. Combin. Theory Ser. A 60 (1992), 143-146. 
[11] F.D. Grosshans, The work of Gian-Carlo Rota on invariant theory, Algebra Universalis 49 (2003), 213-258.

[12] D. Hilbert, Theory of Algebraic Invariants (Cambridge University Press, Cambridge, 1993). Translated from the German and with a preface by Reinhard C. Laubenbacher. Edited and with an introduction by Bernd Sturmfels.

[13] A.N. Kirillov, Unimodality of generalized Gaussian coefficients, C. R. Acad. Sci. Paris Sér. I Math. 315 (1992), 497-501.

[14] K.M. O'Hara, Unimodality of Gaussian coefficients: A constructive proof, J. Combin. Theory Ser. A 53 (1990), 29-52.

[15] I. Pak and G. Panova, Strict unimodality of $q$-binomial coefficients, C. R. Math. Acad. Sci. Paris 351 (2013), 415-418.

[16] I. Pak and G. Panova, Strict unimodality of $q$-binomial coefficients (new version), preprint (2013), arXiv:1306.5085.

[17] I. Pak and G. Panova, Unimodality via Kronecker products, J. Algebraic Combin. 40 (2014), 1103-1120.

[18] I. Pak and G. Panova, Bounds on certain classes of Kronecker and $q$-binomial coefficients, J. Combin. Theory Ser. A 147 (2017), 1-17.

[19] I. Pak, G. Panova and E. Vallejo, Kronecker coefficients: The tensor square conjecture and unimodality, in 26th International Conference on Formal Power Series and Algebraic Combinatorics (FPSAC 2014) (Association of Discrete Mathematics \& Theoretical Computer Science, Nancy, 2014), pp. 149-160.

[20] M. Pouzet and I.G. Rosenberg, Sperner properties for groups and relations, European J. Combin. 7 (1986), 349-370.

[21] R.A. Proctor, Solution of two difficult combinatorial problems with linear algebra, Amer. Math. Monthly 89 (1982), 721-734.

[22] V. Reiner and D. Stanton, Unimodality of differences of specialized Schur functions, J. Algebraic Combin. 7 (1998), 91-107.

[23] G. Salmon, Lessons Introductory to the Modern Higher Algebra, 5th edn. (Cambridge University Press, Cambridge, 1885), reprinted: Bronx, New York, Chelsea Publishing Company.

[24] R.P. Stanley, Weyl groups, the hard Lefschetz theorem, and the Sperner property, SIAM J. Algebraic Discrete Methods 1 (1980), 168-184.

[25] R.P. Stanley, Log-concave and unimodal sequences in algebra, combinatorics, and geometry, in Graph theory and its applications: East and West (Jinan, 1986), Annals of the New York Academy of Sciences, Vol. 576 (New York Academy of Sciences, New York, 1989), pp. 500-535. 
[26] R.P. Stanley, Enumerative Combinatorics, Cambridge Studies in Advanced Mathematics, Vol. 1, 2nd edn. (Cambridge University Press, Cambridge, 2012), p. 49.

[27] R.P. Stanley and F. Zanello, A generalization of a 1998 unimodality conjecture of Reiner and Stanton, J. Comb. 11 (2020), 111-126.

[28] D. Stanton, Unimodality and Young's lattice, J. Combin. Theory Ser. A 54 (1990), 41-53.

[29] B. Sturmfels, Algorithms in Invariant Theory, Texts and Monographs in Symbolic Computation, 2nd edn. (SpringerWienNew York, Vienna, 2008).

[30] J.J. Sylvester, Proof of the hitherto undemonstrated fundamental theorem of invariants, Philos. Mag. 5 (1878), 178-188. Reprinted in: The Collected Mathematical Papers of James Joseph Sylvester, Vol. III (Cambridge University Press, Cambridge, 1909), pp. 117-126.

[31] F. Zanello, Zeilberger's $\mathrm{KOH}$ theorem and the strict unimodality of $q$-binomial coefficients, Proc. Amer. Math. Soc. 143 (2015), 2795-2799.

[32] F. Zanello, On Bergeron's positivity problem for $q$-binomial coefficients, Electron. J. Combin. 25 (2018), Paper No. 2.17, 9 pp.

[33] D. Zeilberger, Kathy O'Hara's constructive proof of the unimodality of the Gaussian polynomials, Amer. Math. Monthly 96 (1989), 590-602. 